\title{
Looking for faces: Attention modulates early occipitotemporal object processing
}

\author{
ANDREAS LUESCHOW, ${ }^{\mathrm{a}}$ TILMANN SANDER,${ }^{\mathrm{a}, \mathrm{b}}$ STEPHAN G. BOEHM, ${ }^{\mathrm{a}}$ GUIDO NOLTE,,${ }^{\mathrm{a}, \mathrm{b}}$ \\ LUTZ TRAHMS, ${ }^{\mathrm{b}}$ AND GABRIEL CURIO ${ }^{\mathrm{a}}$ \\ ${ }^{a}$ Neurophysics Group, Department of Neurology, Campus Benjamin Franklin, Charité-University Medicine, Berlin, Germany \\ ${ }^{\mathrm{b}}$ Physikalisch-Technische Bundesanstalt, Berlin, Germany
}

\begin{abstract}
Looking for somebody's face in a crowd is one of the most important examples of visual search. For this goal, attention has to be directed to a well-defined perceptual category. When this categorically selective process starts is, however, still unknown. To this end, we used magnetoencephalography (MEG) recorded over right human occipitotemporal cortex to investigate the time course of attentional modulation of perceptual processes elicited by faces and by houses. The first face-distinctive MEG response was observed at 160-170 ms (M170). Nevertheless, attention did not start to modulate face processing before $190 \mathrm{~ms}$. The first house-distinctive MEG activity was also found around 160-170 ms. However, house processing was not modulated by attention before $280 \mathrm{~ms}$ ( $90 \mathrm{~ms}$ later than face processing). Further analysis revealed that the attentional modulation of face processing is not due to later, for example, back-propagated activation of the M170 generator. Rather, subsequent stages of occipitotemporal object processing were modulated in a category-specific manner and with preferential access to face processing.
\end{abstract}

Descriptors: Visual attention, Face processing, MEG, M170, Signal-space projection

To select an object in the environment for closer scrutiny, an observer must engage visual attention, either by selecting a position of interest in space, or by the selection of relevant object features. This dichotomous organization (Treisman \& Gelade, 1980) of visual attention has a distinct temporal organization, as revealed in humans by event-related potentials (ERP; Heinze et al., 1994; Hillyard \& Anllo-Vento, 1998; Luck \& Ford, 1998; Mangun, 1995). Spatial attention modulates visual cortical processing as early as 70-100 ms after stimulus onset, whereas for attention directed to elementary features, such as form, color, or motion, ERP modulations start at about $150 \mathrm{~ms}$ (Anllo-Vento, Luck, \& Hillyard, 1998; Harter \& Guido, 1980; Torriente, Valdes-Sosa, Ramirez, \& Bobes, 1999), suggesting that feature selection could occur contingent upon the selection of spatial position (Anllo-Vento \& Hillyard, 1996). These macroscopic ERP findings have their counterpart in single cell recordings. Desimone and colleagues showed for the ventral processing stream in nonhuman primates how attention exerts effects on different levels of extrastriate visual cortex (Chelazzi, Miller, Duncan, \& Desimone, 1993; Luck, Chelazzi, Hillyard, \&

This study has been supported by contracts Ma 1782/1-3,1-4 and GRK 423/1 from the German Research Association (DFG). We acknowledge Walter Endl for kind provision of stimulus materials.

Address reprint requests to: Dr. Andreas Lueschow, Neurophysics Group, Department of Neurology, Campus Benjamin Franklin, CharitéUniversity Medicine, 12200 Berlin, Germany. E-mail: lueschow@zedat. fu-berlin.de
Desimone, 1997; Moran \& Desimone, 1985; Reynolds, Pasternak, \& Desimone, 2000). Analogous effects were shown for single cells in the dorsal processing stream (Recanzone \& Wurtz, 2000; Seidemann \& Newsome, 1999; Treue \& Maunsell, 1996).

Compared to these early attentional modulations of low-level visual processing, the process of selection and identification of complex visual objects is less understood. Face perception can serve as an efficient tool to investigate this operation in more detail. Faces are visually complex objects of primary social importance that require an exquisitely refined identification. They are processed in a specialized area of the human occipitotemporal cortex, the fusiform face area (FFA; Kanwisher, McDermott, \& Chun, 1997; Puce, Allison, Gore, \& McCarthy, 1995; Sergent, Ohta, \& MacDonald, 1992), as shown, for example, by functional magnetic resonance imaging (fMRI), which maps neuronal activations with high spatial resolution through the concomitant neurovascular responses. Specifically, when attention is directed selectively to visual stimuli from one of two different object categories (e.g., faces or houses), the fMRI signal increases in that area of the human occipitotemporal cortex that is specialized for processing of the respective category, for example, the FFA if attention is directed to faces (Clark et al., 1996; Haxby et al., 1994; O’Craven, Downing, \& Kanwisher, 1999; Wojciulik, Kanwisher, \& Driver, 1998).

The present study extends these findings by exploiting the high time resolution of magnetoencephalography (MEG) to determine the earliest latency at which attention influences visual category-specific responses in human occipitotemporal cortex. 
Specifically, it tested the null hypothesis that attention modulates the earliest stages in the visual system that are selectively involved in object processing. In the case of face processing, likely candidates for such attentional modulation are the N170 or its magnetic analogon, the M170.

EEG studies have described the N170 (a negativity occurring bilaterally over occipitotemporal cortex between 150 and $200 \mathrm{~ms}$ ) as the first face-specific response and it has been suggested that this evoked component is the electric counterpart of the process of structural encoding (Bentin, Allison, Puce, Perez, \& McCarthy, 1996; Bentin \& Deouell, 2000; Eimer, 1998, 2000b; Eimer \& McCarthy, 1999).

MEG studies using helmet-shaped sensor systems covering the whole head (Halgren, Raij, Marinkovic, Jousmaki, \& Hari, 2000; Ioannides, Liu, Kwapien, Drozdz, \& Streit, 2000; Liu, Higuchi, Marantz, \& Kanwisher, 2000; Sams, Hietanen, Hari, Ilmoniemi, \& Lounasmaa, 1997; Swithenby et al., 1998; Watanabe, Kakigi, Koyama, \& Kirino, 1999) have convergingly identified a deep neural generator at the fusiform gyrus which (1) is preferentially activated by faces; (2) has a peak response latency around $170 \mathrm{~ms}$, prompting its generic tagging as "M170" generator; (3) can be modeled adequately in most cases as an equivalent current dipole that generates a characteristic magnetic field signature, that is, a bipolar field distribution over the occipitotemporal cortex at the lateral head; and (4) shows a right $>$ left hemisphere preponderance in several studies.

A recent MEG study (Downing, Liu, \& Kanwisher, 2001) showed a modulation of the M170. Subjects were first cued with a stimulus (either a face or a house) and then had to decide whether the cue appeared in a compound stimulus showing a face and a house superimposed. A critical aspect of such compound stimuli is that spatial attention could be used to disambiguate the face and the house at different imaginary depth levels. Thus the modulation of the M170 could be an effect of spatial attention. A partial contribution of spatial attention can also not be ruled out for earlier fMRI studies that used either displays comprising two peripheral faces and two peripheral houses (all presented simultaneously; Wojciulik et al., 1998), or stimuli consisting of a face transparently superimposed on a house (O'Craven et al., 1999).

To exclusively investigate the effect of object-specific attention, the present study used a continuous target detection task where throughout the experimental session only one stimulus at a time was presented to the subjects, always in the center of the visual field, thereby approximating natural viewing conditions during saccadic exploration of the visual environment.

Subjects viewed a random sequence of face and house grayscale photographs. In a block design, either a particular face or a house was the designated target for the following block, and subjects pressed a button upon appearance of the target (Figure 1). By the explicit instruction to attend to the target stimuli, we induced our subjects to attend indirectly also to the nontarget stimuli in the target category as they were naïve with respect to the main focus of the study, which was the analysis of nontarget stimuli.

The contrast of nontarget stimuli (attended vs. unattended) has two advantages: first, it minimizes any possible contribution of motor-related activity to the measured attentional modulation because no response was to be given to the nontarget stimuli. Second, it excludes possible contributions of working memory to the measured attentional modulation because the nontarget stimuli are not maintained in working memory.
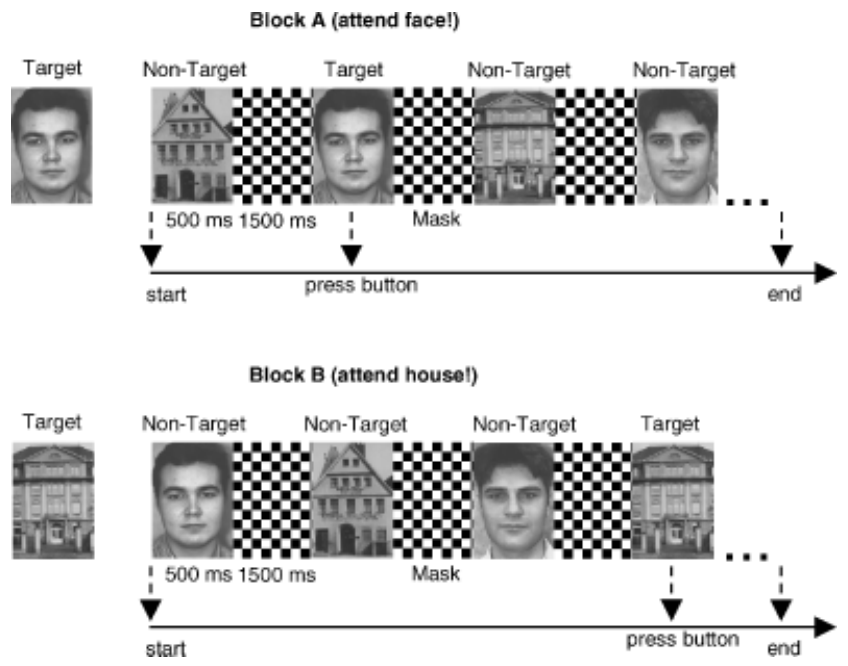

Figure 1. The two blocks of the attention task. Presentation times are indicated.

Simultaneously with MEG, EEG was recorded from nine electrodes referenced against the tip of the nose. This was done for two reasons: (1) MEG is insensitive for radially oriented dipoles. In principle, it is possible that a generator of an attentional modulation has an exclusively radial orientation. We believe that this is practically of little relevance because already a slight deviation from a radial orientation should lead to signal, measurable for MEG. (2) The study should be comparable to EEG work. For example, a recent EEG study with a similar design (Eimer, 2000a) shows an attentional modulation for faces already at $135-180 \mathrm{~ms}$ poststimulus.

Study design and pilot results have been published before in abstract form (Lueschow et al., 2000).

\section{Methods}

\section{Participants}

Ten participants took part ( 5 women, 5 men; all right-handed with normal vision; age range: $20-32$ years). The first 2 participants who only received MEG recordings had to be excluded from the MEG data analysis due to excessive artefacts (muscle and head movements). The remaining 8 participants received simultaneous $\mathrm{MEG} / \mathrm{EEG}$ recordings. All participants were naïve with respect to the experimental purpose. Participants lay supine on a bed, the head turned to the left side and fixed using an evacuated cushion. Each participant gave written informed consent and was compensated for participation. The MEG protocol had been approved before by the local ethics committee (Ethics Committee of the Medical Faculty, Free University Berlin).

\section{Stimuli and Task}

The stimulus set consisted of gray tone images of six faces and six houses that had been digitally scanned. The stimuli were projected through an aperture into the magetically shielded room (Vakuumschmelze Ak 3b, Germany) on a white background. Viewing distance was $0.65 \mathrm{~m}$ and the stimuli subtended a visual angle of $24^{\circ}$ by $24^{\circ}$. Stimulus presentation was controlled by ERTS (Experimental Run Time System, Frankfurt, Germany). 
One picture out of each stimulus class served as the target (Figure 1). The stimuli were shown for $500 \mathrm{~ms}$, followed by a luminance-matched checkerboard mask that stayed on for $1.5 \mathrm{~s}$. During presentation of the mask, a red fixation square was present in the center. The stimuli appeared in random order. Using a block design either a face or a house was the attended target for the following block. Upon appearance of the target participants pressed an optical switch.

The experiment consisted of 24 blocks with 60 stimuli each (25 nontarget face stimuli +5 target face stimuli +25 nontarget house stimuli +5 target house stimuli). Each block had a duration of approximately $2.5 \mathrm{~min}$. The blocks were interdigitated such that participants alternately had to respond to the face target or to the house target. This succession was counterbalanced across participants. The total number of presentations of nontargets is 300 for each of the four conditions (face with or without attention; house with or without attention). At the beginning of the experiment, participants were familiarized with the paradigm and the stimulus set by running four blocks of the experimental session.

\section{Recording}

MEG was recorded in a conventional magnetically shielded room (VAC AK 3b) using a home-made helium cooled SQUID system consisting of 49 axial first-order gradiometers $(70 \mathrm{~mm}$ baseline, $2.7 \mathrm{fT} / \sqrt{ } \mathrm{Hz}$ white noise level), which were arranged in a hexagonal lattice of $30 \mathrm{~mm}$ spacing over a planar area of $210 \mathrm{~mm}$ diameter (Drung, 1995). The dewar was positioned tangentially over the right occipitotemporal cortex, centered over T6 (international 10-20 system). Simultaneously, EEG was recorded from T3, T5, O1, Fz, Cz, Pz, T4, T6, and O2 in 8 of 10 participants (reference electrode at the tip of the nose; impedances below $5 \mathrm{k} \Omega$ ). MEG/EEG data were recorded with a bandpass $0.16-200 \mathrm{~Hz}$ (sampling rate: $500 \mathrm{~Hz}$ ). The data were low-pass filtered off-line at $20 \mathrm{~Hz}$ corner frequency. EOG was measured with two diagonally placed electrodes. Artifact rejection (blinks, eye movements) was performed automatically and removed about $15 \%$ of the recorded trials.

\section{Data Analysis}

The main analysis was carried out on the MEG responses evoked by nontarget objects. Accordingly, four stimulus conditions were compared: responses to nontarget faces when participants directed attention to the target face (FF), nontarget faces during attention to the target house $(\mathrm{FH})$, and the corresponding conditions for nontarget houses $(\mathrm{HH}, \mathrm{HF})$. To identify attentionrelated effects, evoked responses in the unattended condition were subtracted from responses in the attended condition (FF $\mathrm{FH}$; $\mathrm{HH}-\mathrm{HF})$.

\section{Normalized Projection Derived from Signal-Space Projection}

In the signal-space projection (SSP) method (Uusitalo \& Ilmoniemi, 1997) all channels are assumed to be elements of a vector, and the similarity of vectors is measured using the scalar product. We define a normalized projection $p(t)$, which is derived from the SSP: $p(t)=\mathbf{F}(t) \times \mathbf{G} /|\mathbf{G}|^{2}$, where $\mathbf{F}$ and $\mathbf{G}$ are vectors and $\times$ is the scalar product and $|\mathbf{G}|$ the standard vector norm. A measured spatiotemporal pattern is taken as the time-dependent vector $\mathbf{F}(t)$. A time-independent field pattern vector is chosen as $\mathbf{G}$, for example, the measured pattern at $170 \mathrm{~ms}$ in condition $\mathrm{HH}$. The calculated function $p(t)$ monitors then the similarity between the time evolution of the visually evoked fields $\mathbf{F}(t)$ ( $t$ means it varies over time) and the M170 pattern; the normalization $p(t)=1$ for $\mathbf{F}(t=170 \mathrm{~ms})$ enables comparability between different participants. Two different, physiologically motivated choices of $\mathbf{G}$ were employed: (1) at the latency when the M170 upstroke was halfway between onset and peak (showing the M170 pattern fully developed, but with still little contribution from the upcoming attentional signal to the overall signal; range in $n=7$ participants: $150-200 \mathrm{~ms}$ ), (2) at the M230 peak (range: 190-270 ms). Note that the selection of a single/few channels, as often done in the literature, is a projection, too, operating with a binary $(0 / 1)$ channel weighting function.

\section{Results}

\section{MEG Recordings}

Neuromagnetic fields evoked by face or house stimuli were recorded with a planar 49-channel MEG system (Drung, 1995). The planar bottom of the MEG system was centered tangentially at position T6 of the 10-20 EEG system to cover the face processing areas in the right human occipitotemporal cortex. This sensor placement allowed us to chart the typical M170 bipolar field signature (cf. Figure 2a).

For both stimulus categories, two early response components were detected over the right occipitotemporal cortex (Figure 2), peaking for faces on average at $164 \mathrm{~ms}$ (M170) and at $228 \mathrm{~ms}$ (M230), for houses at $156 \mathrm{~ms}$ (M160) and at $252 \mathrm{~ms}$ (M250). The attention-related difference curve for faces (Figure 2a) peaks much earlier $(208 \mathrm{~ms})$ compared to the difference curve for houses (308 ms, Figure 2b). The significance of these attentionrelated difference traces was assessed using a running two-sided $t$ test. For the ensemble of eight individual difference curves, mean amplitude values were calculated in a sliding window (width $60 \mathrm{~ms}$, overlap $30 \mathrm{~ms}$ ) and compared against the mean value in a baseline window ( -60 to $0 \mathrm{~ms} ; p$ values indicated by black squares in Figure 2). The attentional modulation shows a between-category difference in onset latency of $90 \mathrm{~ms}$ (faces: $190 \mathrm{~ms}, p=.029$; houses: $280 \mathrm{~ms}, p=.016$; onset latency defined as center of the first window with $p<.05)$. When we included a Bonferroni correction to the data because of multiple $t$ tests (20 tests in the time range from -0.1 to $0.5 \mathrm{~s}$ ), the threshold of significance fell from .05 to .0025 ; onset latency is then at $220 \mathrm{~ms}$ for face processing and at $310 \mathrm{~ms}$ for house processing, the between-category difference being still $90 \mathrm{~ms}$.

Differences in difficulty do not account for the latency difference of the attentional modulation: Mean reaction times do not differ between categories: $588 \mathrm{~ms}$ for house targets, and $590 \mathrm{~ms}$ for face targets ( $p=.88$; two-tailed paired $t$ test); nor does the error rate although house targets seem to be detected with slightly fewer errors: house targets $4.4 \%$; face targets $6.4 \%$; $p=.2$; two-tailed paired $t$ test.

The onset of the attentional modulation of face processing occurs well after the onset of the M170, identified in earlier studies as face-specific activity, probably generated in the fusiform gyrus (Halgren et al., 2000; Ioannides et al., 2000; Liu et al., 2000; Sams et al., 1997; Swithenby et al., 1998; Watanabe et al., 1999). Despite this difference in onset latency, attention might operate on the very same M170 generator, with top-down activity entering this module simply at a later point in time. This hypothesis was not confirmed when applying SSP (Uusitalo \& Ilmoniemi, 1997); the more technically oriented reader is referred to the Methods section. 

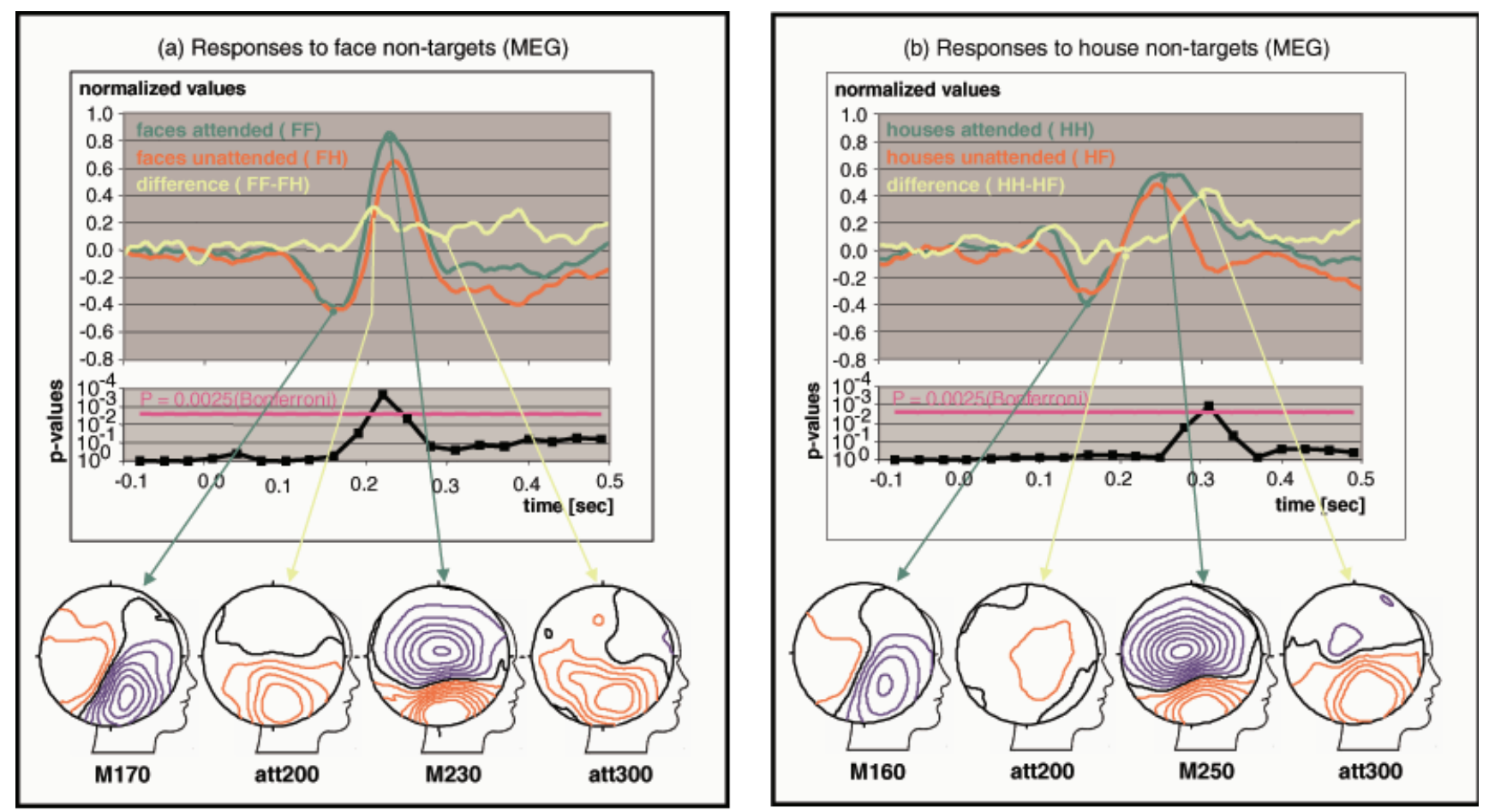

Figure 2. Attention effects for visual MEG responses recorded over the right occipitotemporal cortex: attention directed to faces (large panel in a) or houses (large panel in b); grand average from 8 subjects. Conditions with attention on category of the presented stimulus (green traces: FF and $\mathrm{HH}$ ) are compared to conditions with attention off the stimulus category (red traces: FH and HF). Difference curves extract attention-related effects (yellow traces: FF - FH and HH - HF). The black traces (small panel) indicate the significance level for the yellow difference traces. The pink line represents the level of significance, corrected for multiple comparisons. Below each panel field maps of a representative subject (M.H.) are shown. The first and the third field maps correspond to the peaks of the two early evoked components for faces (M170, M230) and houses (M160, M250). The second and the fourth field maps (labeled att200 and att300) correspond to the peaks of the difference curve for attention to faces or to houses (yellow traces). Before forming the grand average, for each subject a spatial mean had been calculated for the channel with the largest M170/M160 and their six neighbors (the number is due to the hexagonal channel arrangement, cf. Methods section). Then, these original MEG waveformes (showing magnetic field strength in fT) had been normalized for each subject with respect to the M230 of condition FF.

The logic of this approach is qualitatively illustrated as follows: Suppose the attentional modulation that onsets at $190 \mathrm{~ms}$ is due to later activation of the M170 generator, for example, by back-projections. In this case, there should be significant if not total overlap between the neuronal ensemble that constitutes the M170 generator and the neuronal ensemble that constitutes the generator of the attentional modulation. The problem with the difference curves that describe the evolution of the attentional modulation is that at any point in time, multiple generators contribute to the measured signal. As a consequence, inspection of the curve does not give the answer to the question of which generator contributes significantly and which one does not.

To solve this problem of multiple generators that are simultaneously active we applied SSP. SSP was used to maximize selectively the contribution of two obviously separate generators that underlie the first two evoked components in our data, namely the M170 and the M230 and to test which one contributes to the attentional modulation and which one does not.

For the resulting curves, the same running $t$ test was applied as for the original difference curves (attended minus unattended stimuli). The difference curve with maximized contribution from the M170 (dashed line of Figure 3) shows no statistically significant modulation whereas the difference curve with maximized contribution from the M230 (continuous line of Figure 3) shows a significant modulation around $200 \mathrm{~ms}$. A straightforward interpretation is that the M230 module does significantly contribute to the attentional modulation whereas the M170 does not. In other words, attention does not operate on the generator of the M170 but on subsequent stages of processing.

The general result for the group is evident by visual inspection of the field pattern of subject M.H. in Figure 2a, the bipolar field pattern at the peak of the evoked M170 component has an oblique orientation obviously different from the horizontal pattern orientation at the peak of the M230 component.

\section{Analysis of Target Stimuli}

The main purpose of this experiment was to study MEG responses to nontarget objects that are expected to be less contaminated by motor-related activity and working memory. The paradigm was thus designed so as to yield a high signal-tonoise ratio for the nontargets, that is, 300 trials in each of the conditions (cells) of the $2 \times 2$ factorial design (FF, FH, HH, $\mathrm{HF}$ ). The target stimuli were shown with a frequency of one out of six (cf. Methods section) which gives 60 trials per condition. The relevant conditions are called: $\mathrm{FF}^{\mathrm{T}}$ (face targets attended), 


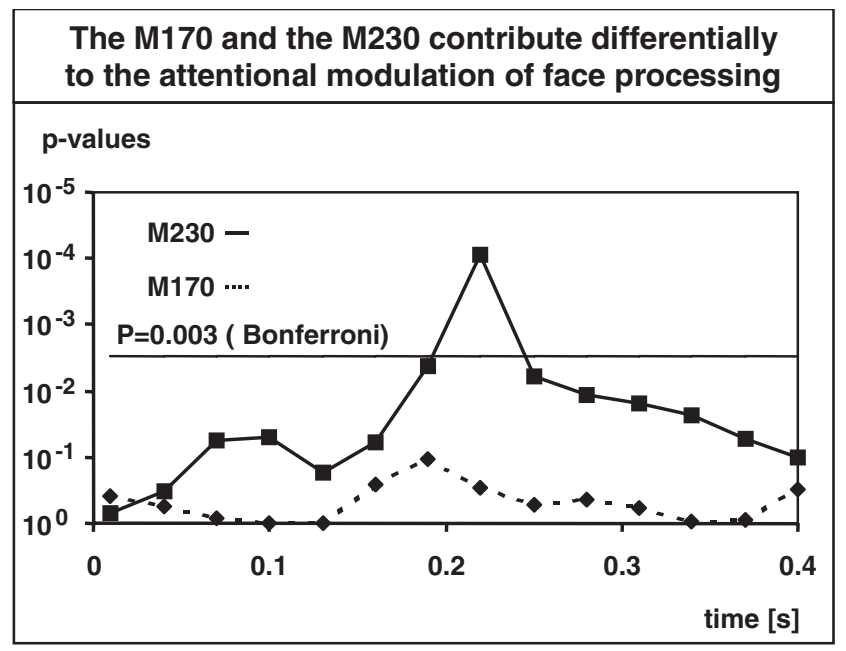

Figure 3. Significance of attention-related neuronal activations as a function of latency poststimulus. Depicted are levels of significance from running $t$ tests that compare the attention-related difference curves (faces attended minus faces unattended) against prestimulus baseline. The continuous trace represents the attention related difference curve with "maximized" contribution of the M230; the dashed line represents the attention-related difference curve with "maximized" contribution of the M170. Higher significance indicates that a component is more similar to the attention-related field pattern, that is, "it carries more of the attentional effect" observed in the evoked magnetic response with contribution of all possible components. The horizontal black line represents the level of significance, corrected for multiple comparisons.

$\mathrm{FH}^{\mathrm{T}}$ (face targets unattended), and $\mathrm{HH}^{\mathrm{T}}$ and $\mathrm{HF}^{\mathrm{T}}$ for the house targets, respectively. As expected, due to the lower number of conditions, conventional averaging gave very poor results with a low signal-to-noise ratio and no clear indication of an early attentional effect for face stimuli.

To remove stimulus-unrelated background activity (alphawaves and cardiac artifacts), we applied independent component analysis (ICA). The method can reduce substantially the number of averages that are needed to obtain a reasonable signal-to-noise ratio in cognitive MEG data (Sander, Wübbeler, Lueschow, Curio, \& Trahms, 2002).

Figure 4 shows a grand average for 7 subjects. As for nontargets, two early responses are obtained for both categories, at $165 \mathrm{~ms}$ and $236 \mathrm{~ms}$ for faces (M170, M230) and at 188 and $266 \mathrm{~ms}$ for houses (M160, M250), which do not systematically differ from the nontarget responses given the overall lower signalto-noise ratio that is evident in Figure 4.

The time course of the attentional modulation for target stimuli is almost identical to the nontarget stimuli: an early modulation of face processing that peaks at $196 \mathrm{~ms}$ (nontarget stimuli $208 \mathrm{~ms}$ ). As for nontarget stimuli, there is no early attentional modulation of house processing for target stimuli. The attentional modulation of house processing shows a broad peak between 340 and $350 \mathrm{~ms}$. The significance of the attentionrelated difference traces was evaluated using the same running $t$ test as for the nontarget data of Figure 2a,b (cf. above). Onset latency, defined as center of the first window with $p<.05$ is $220 \mathrm{~ms}$ for face processing, $p=.019$, and $310 \mathrm{~ms}$ for house processing, $p=.045$. For target stimuli of both categories, onset latency is $30 \mathrm{~ms}$ longer compared to nontarget stimuli, which is presumably due to the lower signal-to-noise ratio.
Responses to face targets (MEG)

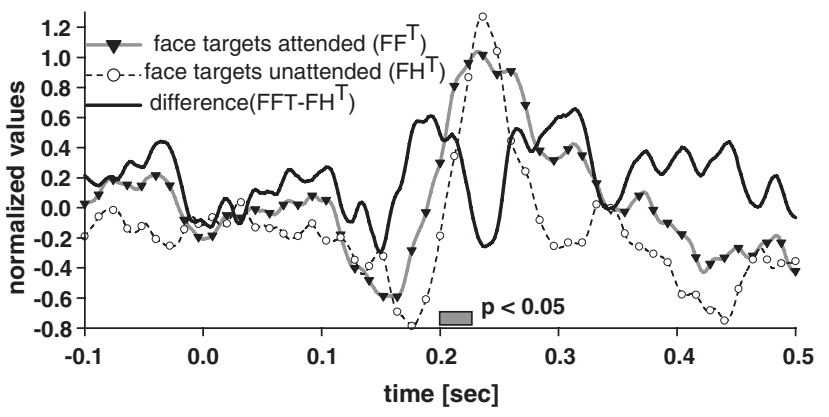

Responses to house targets (MEG)

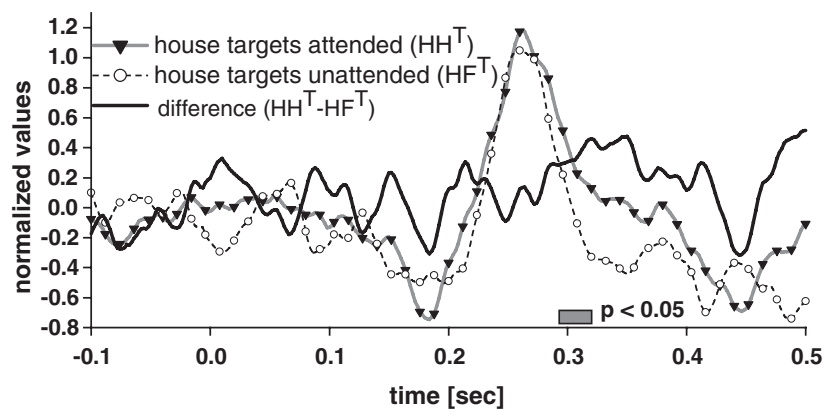

Figure 4. MEG responses to target stimuli (upper panel: face targets; lower panel: house targets). Grand average from 7 subjects. For each subject the same spatial mean was calculated as in Figure 2a,b. The gray bars mark the intervals when the attention-related difference curves (black lines) differ from the base line at $p<.05$ ( $t$ test).

\section{EEG Recordings}

Simultaneously to MEG, EEG was recorded from nine electrodes (cf. Figures 5 and 6 and Methods section) referenced against the tip of the nose. For faces, two deflections can be seen at occipital and temporal sites, the first being an initial negativity with a peak between 140 and $150 \mathrm{~ms}$, presumably corresponding to the well-described N170 (e.g., Bentin et al., 1996). The second deflection is a broader positivity peaking around $230 \mathrm{~ms}$ (here P230). At midline electrodes, three deflections are present: first, a positivity around $170 \mathrm{~ms}$, maximal at $\mathrm{Cz}$, probably the $\mathrm{P} 150$ (Botzel \& Grusser, 1989; Jeffreys, 1989); second, a negativity with a peak at $230 \mathrm{~ms}$; and third, a broader positivity with a peak at $300 \mathrm{~ms}$. No lateralization was evident. For houses, at midline electrodes similar deflections with similar peak latencies are obtained as for faces, which indicates that they are caused by overlapping or identical neural sources. At lateral sites, the picture for houses is quite different from that for faces. As for faces, an initial negative deflection with a peak between 140 and $150 \mathrm{~ms}$ is present. But in contrast to face stimuli, the positive deflection with a peak at $230 \mathrm{~ms}$ is lacking; instead a broad negativity with a dip around $300 \mathrm{~ms}$ is present. In addition, EEG, like MEG recordings, are lacking early responses around $100 \mathrm{~ms}(\mathrm{P} 1)$.

In comparison to Figures 5 and 6 in which the pink bars indicate the area where the attention-related difference curves differ from the baseline at $p<.0025$ (significance threshold corrected for multiple comparisons) Table 1 shows the onsets of the attentional modulations for face and house processing defined as the first window in the running $t$ test with $p<.05$. 
Responses to face non-targets (EEG)

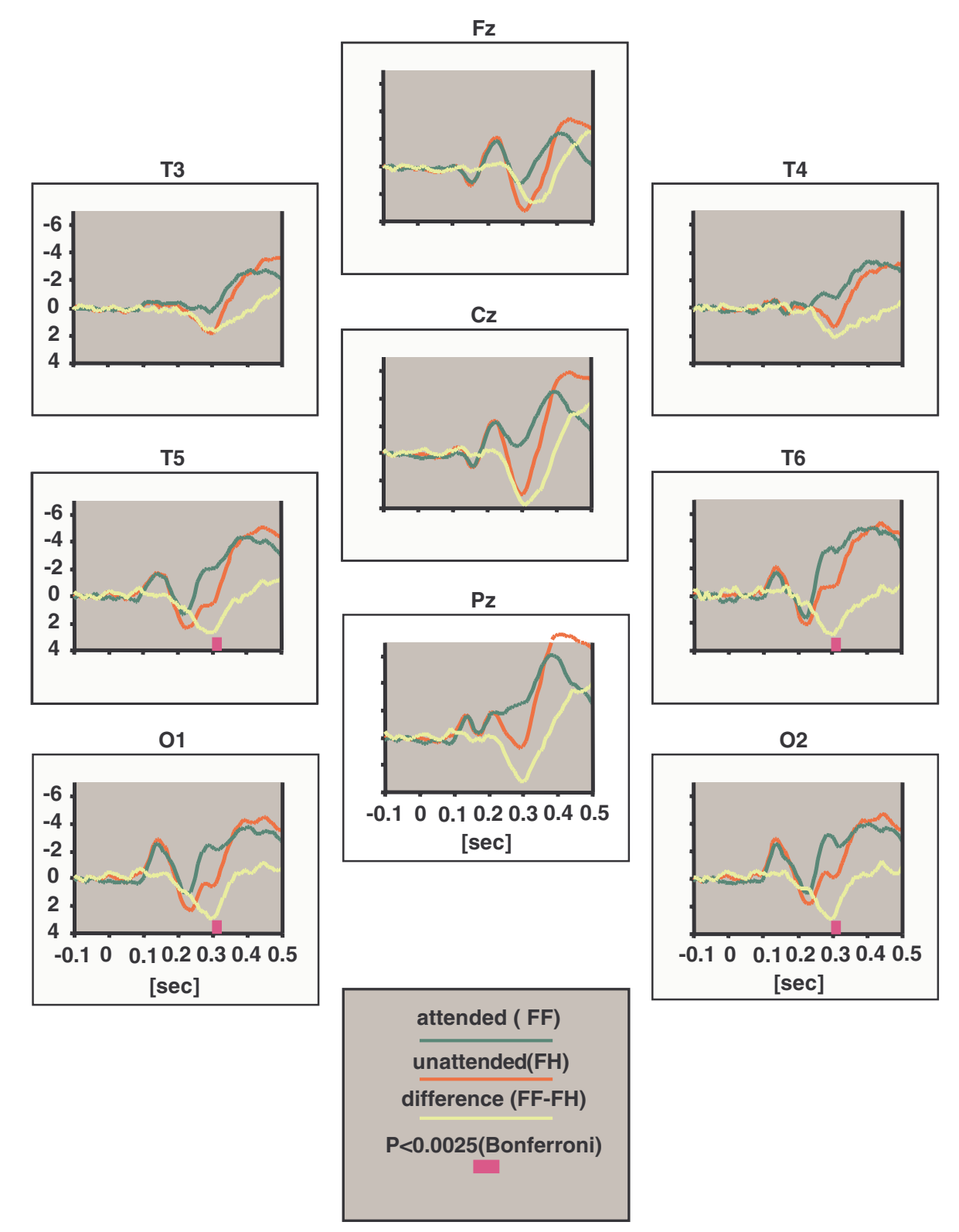

Figure 5. EEG (from eight electrodes of the 10-20-system; grand averages from 8 subjects) for face stimuli showing the main attention-related EEG modulations. Trace color codes as in Figure 2. The pink bars mark the intervals when the attention-related difference curves (yellow lines) differ from the base line at $p<.0025$ (level of significance corrected for multiple comparisons).

Also, with this less conservative criterion there is no systematic difference between the two categories and between hemispheres, with most onset latencies lying between 280 and $310 \mathrm{~ms}$. The onset latencies for houses are shortest at O1 and T5 at $250 \mathrm{~ms}$. Interestingly, the onset latencies detected by EEG coincide with the late attentional modulation as detected by MEG for faces and houses. But, most importantly, EEG, like MEG, does not detect any early attentional effect for houses, excluding the possibility that a radially oriented dipole for which MEG would be insensitive could carry that information.

\section{Late Attentional Effects (Selection Negativity)}

Besides the early modulation of face processing that peaks at $200 \mathrm{~ms}$ and is only detected by MEG, there is a later attentional modulation that starts around $250 \mathrm{~ms}$ and peaks between 300 and $350 \mathrm{~ms}$. We wondered whether this component, the time course of which resembles the selection negativity (e.g., AnlloVento \& Hillyard, 1996; Harter \& Guido, 1980; Harter, Aine, \& Schroeder, 1982; Hillyard \& Münte, 1984), indexes different category-related cortical generators or whether one single "all purpose" attentional processor is differentially active in case of house or face processing due to, for example, mere differences in task difficulty. Therefore we calculated the similarity between MEG field maps, again using a normalized projection (cf. also Methods and Results sections).

The normalized projection can be interpreted as a spatial correlation between two field maps, and low or negative correlations correspond to different maps and this corresponds 


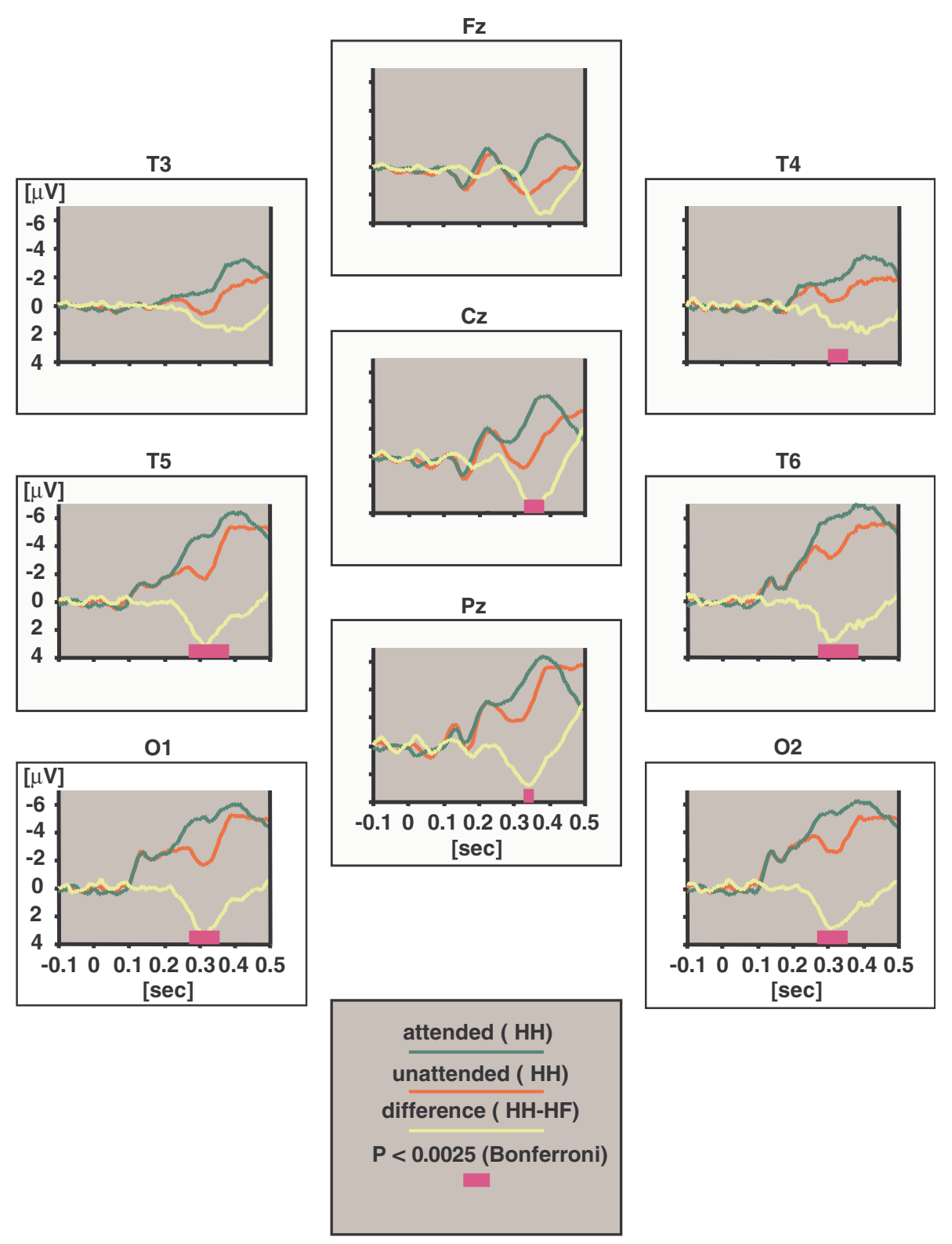

Figure 6. EEG (from eight electrodes of the 10-20-system; grand averages from 8 subjects) for house stimuli showing the main attention-related EEG modulations. Trace color codes as in Figures 2 and 5.

to different sources. This approach is different from the one widely used by ERP researchers to evaluate differences of field topographies. Usually it is inferred from a significant Electrode $\times$ Condition interaction that two field topographies are different, which is then interpreted as being indicative of different "spatial" structure of the underlying source(s).

To avoid "artificially" significant interactions due to simple changes in source strength McCarthy and Wood (1985) recommended scaling or normalizing the data, for example, by vector length, prior to statistical testing. Notwithstanding critical evaluations (Haig, Gordon, \& Hook, 1997; Ruchkin, Johnson, \& Friedman, 1999; Urbach \& Kutas, 2002), normalization procedures are widely used and recommended in ERP guidelines (Picton et al., 2000).
Because the present coarse EEG electrode setting does not allow analysis of field topographies, we use the MEG difference patterns between houses attended and houses unattended $\mathrm{(HH}$ - HF) and faces attended and unattended (FF - FH). By visual inspection we identified clearly structured patterns for both categories around $300 \mathrm{~ms}$ individually, which coincided with significant differences in individual $t$ tests for the channel with the largest response. In one subject, no late attentional effect for faces could be detected and it was excluded from the analysis.

For comparing two different field maps, the normalized projection reads $\operatorname{proj}=(\mathbf{F} /|\mathbf{F}|) \times(\mathbf{G} /|\mathbf{G}|)$, where $\mathbf{F}$ and $\mathbf{G}$ are, for example, the maps of the late attentional effect for faces and houses, respectively. Note that we use a normalization by vector length. For identical patterns $\mathbf{F}$ and $\mathbf{G}$ the proj value becomes 1 , and $\operatorname{proj}=0$ 
Table 1. Onset of Late Attentional Modulation for Faces and Houses in EEG, $p<.05$

\begin{tabular}{|c|c|c|c|c|c|c|c|c|c|}
\hline & \multicolumn{9}{|c|}{ Electrode } \\
\hline & $\mathrm{O} 1$ & $\mathrm{O} 2$ & T5 & T6 & $\mathrm{T} 3$ & $\mathrm{~T} 4$ & $\mathrm{Pz}$ & $\mathrm{Cz}$ & $\mathrm{Fz}$ \\
\hline \multicolumn{10}{|l|}{ Faces } \\
\hline Latency (ms) & 280 & 280 & 280 & 280 & 340 & 310 & 310 & 310 & 310 \\
\hline$p$ value & .017 & .02 & .036 & .03 & .047 & .017 & .025 & .009 & .019 \\
\hline \multicolumn{10}{|l|}{ Houses } \\
\hline Latency (ms) & 250 & 280 & 250 & 280 & 310 & 310 & 280 & 310 & 340 \\
\hline$p$ value & .008 & .0005 & .01 & .0009 & .036 & .0009 & .037 & .019 & .03 \\
\hline
\end{tabular}

for orthogonal patterns. A proj $=-1$ identifies current sources having a similar location but with an opposite primary current.

For the 7 subjects that entered the analysis, the calculation results in an average proj value of .56 (SD of .44). This is midway between identical and orthogonal, and it indicates that the underlying neuronal populations relevant for the attentional effects are not identical. This was statistically evaluated for the group of 7 subjects by a paired $t$ test that tested whether the difference between the individual proj value and a proj value of 1 (identical patterns) differs significantly from zero, $t(6)=2.697$, $p=.036$ (two-sided).

The difference for the group is exemplified on a showcase in Figure 7. For this example $p=.1$ results at $t=300 \mathrm{~ms}$ as the maps are almost orthogonal.

\section{Discussion}

\section{Attention Has a Preferred Access to Face Processing}

In this $\mathrm{MEG} / \mathrm{EEG}$ study, top-down attentional control has been shown to operate in a category-specific manner on the processing of complex visual objects in human occipitotemporal cortex. This complements recent PET (Haxby et al., 1994) and fMRI work (Clark et al., 1996) that demonstrated that attending to faces (as opposed to locations or color) causes an increase of activity in areas associated with face processing in occipitotemporal cortex. Generally, object-specific activity, in the fusiform gyrus for faces

Difference maps for faces and houses (attended minus unattended)

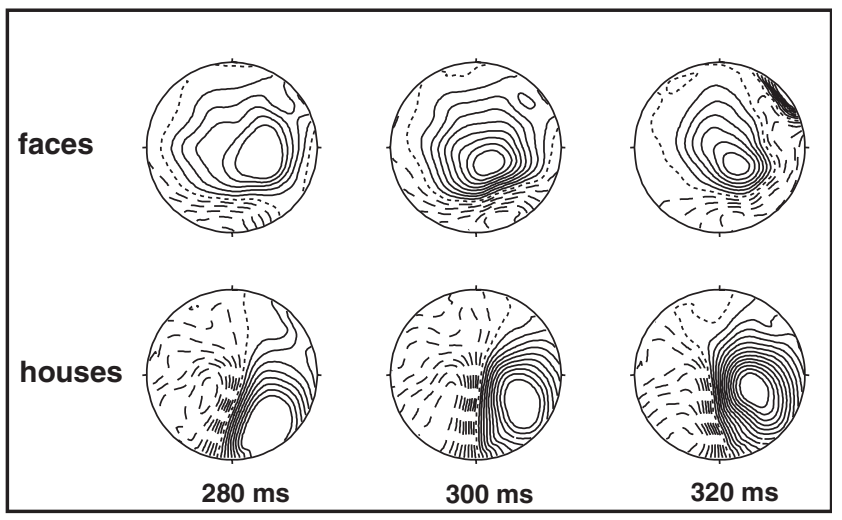

Figure 7. Difference maps for faces and houses (attended minus unattended) at three points late in time showing relative stable field topographies. The normalized projection was calculated at $300 \mathrm{~ms}$, which coincided with the peak of the late attentional effect in the difference curves.
(Wojciulik et al., 1998) or in the parahippocampal place area for the processing of houses (Aguirre, Zarahn, \& D'Esposito, 1998; Epstein \& Kanwisher, 1998), was stronger when the corresponding visual stimulus was the attended one (O'Craven et al., 1999).

The present MEG data provide essential new information on the time course of these effects: Face processing starts to be modulated by attention as early as $190 \mathrm{~ms}$, whereas the onset of a significant attentional modulation of house processing occurs $90 \mathrm{~ms}$ later at $280 \mathrm{~ms}$. The contrast of nontarget stimuli (attended vs. unattended) as realized here minimizes any possible contribution of motor-related activity to the measured attentional modulation because no response was to be given to the nontarget stimuli. Thus, top-down control has a preferred fast access to the processing of faces, which represent an overlearned and socially important stimulus class.

Our data make it unlikely that faster categorization for faces accounts for the earlier onset of the attentional modulation for faces compared to houses. The M230 response occurs just $20 \mathrm{~ms}$ before the M250 response. This difference of $20 \mathrm{~ms}$ does not account fully for the much larger difference of the onset of the attentional modulation, which is $90 \mathrm{~ms}$.

Also, differences in difficulty in detecting face versus house targets do not account for the latency difference of the attentional modulation because reaction times and error rates were not significantly different between the two tasks.

\section{The Occipitotemporal M-170 Generator Is Not Modulated by Attention}

In close agreement with a recent MEG study (Halgren et al., 2000), the face-specific component of the evoked response peaked at $164 \mathrm{~ms}$ ("M170"). Because of the present block design, a sustained biasing of early face processing could be expected, with the effect that attention operates upon the earliest face-related response. Interestingly and unexpectedly, the attentional modulation of face processing did not start before $190 \mathrm{~ms}$. Still, reentrant activity could modulate the cell assembly underlying the M170 at some later point in time. Then the field distribution generated by the attentionally modulated neuronal ensemble should contain a significant contribution from the mainly dipolar M170 field distribution. However, using SSP (Uusitalo \& Ilmoniemi, 1997) to selectively maximize the contribution of two obviously separate generators that underlie the first two evoked components in our data, namely the M170 and the M230, showed that the M170 generator does not significantly contribute to the attentional modulation whereas the M230 generator does significantly contribute. Hence, attention modulates stages of processing that lie downstream to the occipitotemporal M170 generator (Halgren et al., 2000; Ioannides et al., 2000; Liu et al., 2000; Sams et al., 1997; 
Swithenby et al., 1998; Watanabe et al., 1999), which represents an early, rather automatic stage in face processing.

Given the spatial overlap of the M170 field and the attentionrelated difference field, we suggest that the functional unit for attentional modulation is localized in the vicinity of the M170 generator in the occipitotemporal cortex. Additional spatial analyses using multisource modeling, however, proved to be unstable, mainly because of this sensor's relatively focal view. In place of such a source analysis, which has to refer back to both source and volume conductor modeling, a model-free projection approach was chosen here. This exploits the full dimensionality of the available signal space (as defined by the number of sensors) but, advantageously, does not rely on other model assumptions.

Notably, the M170 is a compound signal: Watanabe and colleagues (Watanabe et al., 1999; Watanabe, Kakigi, \& Puce, 2003) modeled early face-related activity by two different dipoles, and complementary fMRI showed activation of different spots (e.g., Hoffman \& Haxby, 2000). Functionally, the M170 also confounds face-specific activity with activity that is not domain specific. By using SSP to maximize contributions from this M170, the present study found no attentional modulation. Thus, possible M170 subcomponents (contributing significantly to the direction of the M170 compound vector) also appeared unmodulated, so that it is unlikely that a face-specific subcomponent is modulated by attention via reentrant processing.

Because of its similar latency, the M170 in the present study corresponds to the earliest face-sensitive component characterized in other MEG or EEG studies (e.g., Bentin et al., 1996; Halgren et al., 2000). Functionally the M170 relates to the stage of structural encoding (e.g., Bentin \& Deouell, 2000) and our attention task, consistent with the literature (e.g., Bentin \& Deouell, 2000), adds to the evidence so far that this component is impervious to top-down processing.

The relation of the M230 to previous ERP work is less clear. Functionally, we would relate it to the stage of face recognition units (Bruce \& Young, 1986), preexistent face representations that can be activated by top-down processing.

\section{Is the Relatively Late Attentional Modulation of Face Processing Due to Low Perceptual Load?}

Within the debate of early and late selection theories of attention, it has been argued that perceptual load is a major determinant of the locus of selection (Lavie, 1995; Lavie \& Tsal, 1994): If perceptual load is high, selection occurs early and vice versa. Perceptual load is determined by different factors, for example, display size, presentation time, and presentation rate. With respect to the latter two factors, perceptual load in the present paradigm is rather low, and it could be argued that only because of this does attentional modulation of face processing occur relatively late, that is, after the M170. This is indeed conceivable but it was not the point of this study.

We intended to determine the stage upon which attention acts if faces are selected by their Gestalt (their "faceness") because this, at the same time, clarifies the issue when in the sequence of visual processing specialized face processing takes place for the first time. Because of this goal we had to keep perceptual load low to allow subjects to select faces "at a glance" (i.e., holistically) as they naturally tend to do because of training by long experience and by everyday social interaction. It is, of course, conceivable that in case of increased perceptual load, faces had been selected by a simpler more local feature such as a mole on the cheek indexed by an earlier ERP component, but this was not our intention.
Possible Relation of the Attentional Modulation to the N200

The early attentional modulation of face processing peaks at $208 \mathrm{~ms}$. This suggests a possible link to the face-specific N200 characterized in intracranial recordings from occipitotemporal cortex (Allison, Puce, Spencer, \& McCarthy, 1999; McCarthy, Puce, Belger, \& Allison, 1999; Puce, Allison, \& McCarthy, 1999). However, this N200 was not susceptible to top-down influences, such as semantic priming. Because no tests specific for selective attention were employed, it remains an open question whether the intracranial N200 can be modulated by attention. Interestingly, the N200 to another nonface category (words; Nobre, Allison, \& McCarthy, 1998) similar to houses in the present study was found to be impervious to attentional manipulation.

\section{Targets Are Not Processed Differently from Nontargets}

One could argue that attending directly to the rare targets (subjects were explicitly instructed to attend to the targets) could involve a qualitatively different attentional modulation than the indirect orienting to the nontarget stimuli. For example, because the targets are exclusively maintained in working memory, this could result in a sustained biasing only for the processing of these stimuli, with the effect that only for target stimuli would the M170 module be modulated. This possibility is rendered unlikely given that the time course of the attentional modulation of face and house targets is similar to the attentional modulation of the processing of nontargets. Especially, also for face targets, the early attentional modulation peaks well after the peak of the M170. In principle, a minor effect might remain camouflaged due to the lower signal-to-noise ratio of the target data, but there is no indication for such a possibility in Figure 4.

\section{Late Attentional Effects Are Specific for Face and House Processing}

Besides the early modulation of face processing that is only detected by MEG and peaks at $200 \mathrm{~ms}$, EEG and MEG detected both for face and house processing a later attentional modulation that starts between 200 and $250 \mathrm{~ms}$ and peaks between 300 and $350 \mathrm{~ms}$. This component resembles the selection negativity (SN), which indexes the selection of features (e.g., Anllo-Vento \& Hillyard, 1996; Harter \& Guido, 1980; Harter et al., 1982; Hillyard \& Münte, 1984). It starts between 150 and $200 \mathrm{~ms}$, is contingent upon prior selection of location, and its field topography changes depending on the type of feature being selected. The latter indicates that category-specific cortices can be independently modulated by attention. Our analysis of field topograpies shows that they are statistically different for the group as a whole, which indicates that it is not only at the earlier stage that attentional processing of faces and houses is qualitatively different - that is, has a different neural substrate-but also at this later stage. It should be stressed here that the field topography itself and the degree of similarity changes markedly interindividually. Therefore we consider it inappropriate to compute grand averages of field topographies. Eimer (2000a), in his EEG recordings, observes a very similar component starting around $200 \mathrm{~ms}$ and peaking around $300 \mathrm{~ms}$ that he considers as category unspecific because of a similar distribution over his electrode array. Such a conclusion is premature in our opinion because of the coarse electrode setting Eimer used. The grand-averaged EEG in this experiment with the exact same setting and referencing as in Eimer's study did not show a difference for the late attentional effect either, whereas MEG with its denser array of channels detected a clear-cut difference in topograhy. 


\section{Earlier Findings Concerning the Attentional Modulation of Face Processing}

A recent MEG study (Downing et al., 2001) showed a modulation of the M170. As was pointed out already in the Introduction, due to the use of a compound stimulus (a face transparently superimposed on a house) in this study, a contribution of spatial attention cannot be ruled out. Second, in the experiment, a single stimulus, a face or a house cue, was shown $800 \mathrm{~ms}$ in advance of the compound stimulus and the subjects had to indicate whether the single stimulus appeared in the compound stimulus. Therefore it could be that the observed amplification of the M170 is an effect of the cue on the compound stimulus and not due to attentional modulation. The authors argue that repetition should reduce the amplitude of the evoked response, but recent fMRI work shows that depending on the task repetition can also enhance the amplitude of the BOLD response (Henson, Shallice, \& Dolan, 2000).

An EEG study (Eimer, 2000a) showed an even earlier attentional modulation for faces at $135-180 \mathrm{~ms}$ poststimulus. This might reflect modulation of lower level feature processing (upstream to face-specific cortical areas) because the nonface stimuli employed (chairs) appear to differ substantially from the face stimuli, for example, with respect to spatial frequencies. This is less obvious for the stimuli used here. A second reason why we do not observe this early attentional modulation in our MEG and EEG recordings probably is that the low level mechanisms are "saturated" because one structured pattern (a face or a house) is followed by another structured pattern (a luminance adapted checkerboard mask). The fact that we observe no P100 after onset of faces or houses indicates that the low level mechanisms are indeed saturated. In contrast, Eimer's stimuli were followed by a white homogeneous background.

A third study using EEG did not find an early attentional effect (in the time range from 100 to $250 \mathrm{~ms}$ ) when attention was switched inside one category (faces with eyes closed and eyes open; Cauquil, Edmonds, \& Taylor, 2000). Such stimuli drive neuronal ensembles with a high degree of overlap, rendering a possible attentional effect indiscernible in the evoked response. Notably, substantial overlap of fMRI activations has been shown recently even for stimuli as distinct as faces, houses, and chairs (Haxby et al., 2001; Ishai, Ungerleider, Martin, Schouten, \& Haxby, 1999).

\section{Conclusion}

Attention modulates early visual object processing in a categoryspecific manner. Top-down control has a preferred fast access to face processing in the human right occipitotemporal cortex; however, the very first stage of face processing at about $170 \mathrm{~ms}$ is not modulated by category-specific attention.

Thus we favor an attentional framework where objects can be selected on the basis of position in space and/or features depending on behavioral demands. The speed of selection of an object depends on the level of the visual hierarchy where the property is coded on which the selection is based. In such a framework, selecting space seems to be the fastest process, followed by the selection of simpler features like color or form. In the present experiment where subjects select objects on the basis of their Gestalt or Gestalt features, the selection process takes even longer and its late timing sheds light on the hierarchy of visual processing.

\section{REFERENCES}

Aguirre, G. K., Zarahn, E., \& D'Esposito, M. (1998). An area within human ventral cortex sensitive to building stimuli: Evidence and implications. Neuron, 21, 373-383.

Allison, T., Puce, A., Spencer, D. D., \& McCarthy, G. (1999). Electrophysiological studies of human face perception. I: Potentials generated in occipitotemporal cortex by face and non-face stimuli. Cerebral Cortex, 9, 415-430.

Anllo-Vento, L., \& Hillyard, S. A. (1996). Selective attention to the color and direction of moving stimuli: Electrophysiological correlates of hierarchical feature selection. Perception \& Psychophysics, 58, 191-206.

Anllo-Vento, L., Luck, S. J., \& Hillyard, S. A. (1998). Spatio-temporal dynamics of attention to color: Evidence from human electrophysiology. Human Brain Mapping, 6, 216-238.

Bentin, S., Allison, T., Puce, A., Perez, E., \& McCarthy, G. (1996). Electrophysiological studies of face perception in humans. Journal of Cognitive Neuroscience, 8, 551-565.

Bentin, S., \& Deouell, L. Y. (2000). Structural encoding and identification in face processing: ERP evidence for separate mechanisms. Cognitive Neuropsychology, 17, 35-54.

Botzel, K., \& Grusser, O. J. (1989). Electric brain potentials evoked by pictures of faces and non-faces: A search for face-specific EEGpotentials. Experimental Brain Research, 77, 349-360.

Bruce, V., \& Young, A. W. (1986). Understanding face recognition. British Journal of Psychology, 77, 305-327.

Cauquil, A. S., Edmonds, G. E., \& Taylor, M. J. (2000). Is the facesensitive N170 the only ERP not affected by selective attention? Neuroreport, 11, 2167-2171.

Chelazzi, L., Miller, E. K., Duncan, J., \& Desimone, R. (1993). A neural basis for visual search in inferior temporal cortex. Nature, $363,345-$ 347.

Clark, V. P., Keil, K., Maisog, J. M., Courtney, S., Ungerleider, L. G., \& Haxby, J. V. (1996). Functional magnetic resonance imaging of human visual cortex during face matching: A comparison with positron emission tomography. Neuroimage, 4, 1-15.
Downing, P., Liu, J., \& Kanwisher, N. (2001). Testing cognitive models of visual attention with fMRI and MEG. Neuropsychologia, 39, 13291342.

Drung, D. (1995). The PTB 83-SQUID-system for biomagnetic applications in a clinic. IEEE Transactions on Applied Superconductivity, 5, 2112-2117.

Eimer, M. (1998). Does the face-specific N170 component reflect the activity of a specialized eye processor? Neuroreport, 9, 2945-2948.

Eimer, M. (2000a). Attentional modulations of event-related potentials sensitive to faces. Cognitive Neuropsychology, 17, 103-116.

Eimer, M. (2000b). Effects of face inversion on the structural encoding and recognition of faces. Evidence from event-related brain potentials. Brain Research Cognitive Brain Research, 10, 145-158.

Eimer, M., \& McCarthy, R. A. (1999). Prosopagnosia and structural encoding of faces: Evidence from event-related potentials. Neuroreport, 10, 255-259.

Epstein, R., \& Kanwisher, N. (1998). A cortical representation of the local visual environment. Nature, 392, 598-601.

Haig, A. R., Gordon, E., \& Hook, S. (1997). To scale or not to scale: McCarthy and Wood revisited. Electroencephalography and Clinical Neurophysiology, 103, 323-325.

Halgren, E., Raij, T., Marinkovic, K., Jousmaki, V., \& Hari, R. (2000). Cognitive response profile of the human fusiform face area as determined by MEG. Cerebral Cortex, 10, 69-81.

Harter, M. R., \& Guido, W. (1980). Attention to pattern orientation: Negative cortical potentials, reaction time, and the selection process. Electroencephalography and Clinical Neurophysiology, 49, 461-475.

Harter, R. M., Aine, C., \& Schroeder, C. (1982). Hemispheric differences in the neural processing of stimulus location and type: Effects of selective attention on visual evoked potentials. Neuropsychologia, 20, 421-438.

Haxby, J. V., Gobbini, M. I., Furey, M. L., Ishai, A., Schouten, J. L., \& Pietrini, P. (2001). Distributed and overlapping representations of faces and objects in ventral temporal cortex. Science, 293, 2425-2430. 
Haxby, J. V., Horwitz, B., Ungerleider, L. G., Maisog, J. M., Pietrini, P., \& Grady, C. L. (1994). The functional organization of human extrastriate cortex: A PET-rCBF study of selective attention to faces and locations. Journal of Neuroscience, 14, 6336-6353.

Heinze, H. J., Mangun, G. R., Burchert, W., Hinrichs, H., Scholz, M., Munte, T. F., Gos, A., Scherg, M., Johannes, S., Hundeshagen, H., Gazzaniga, M. S., \& Hillyard, S. A. (1994). Combined spatial and temporal imaging of brain activity during visual selective attention in humans. Nature, 372, 543-546.

Henson, R., Shallice, T., \& Dolan, R. (2000). Neuroimaging evidence for dissociable forms of repetition priming. Science, 287, 1269-1272.

Hillyard, S. A., \& Anllo-Vento, L. (1998). Event-related brain potentials in the study of visual selective attention. Proceedings of the National Academy of Sciences, USA, 95, 781-787.

Hillyard, S. A., \& Münte, T. F. (1984). Selective attention to color and location: An analysis with event-related potentials. Perception \& Psychophysics, 36, 185-198.

Hoffman, E. A., \& Haxby, J. V. (2000). Distinct representations of eye gaze and identity in the distributed human neural system for face perception. Nature Neuroscience, 3, 80-84.

Ioannides, A. A., Liu, L. C., Kwapien, J., Drozdz, S., \& Streit, M. (2000). Coupling of regional activations in a human brain during an object and face affect recognition task. Human Brain Mapping, 11, $77-92$.

Ishai, A., Ungerleider, L. G., Martin, A., Schouten, J. L., \& Haxby, J. V. (1999). Distributed representation of objects in the human ventral visual pathway. Proceedings of the National Academy of Sciences, USA, 96, 9379-9384.

Jeffreys, D. A. (1989). A face-responsive potential recorded from the human scalp. Experimental Brain Research, 78, 193-202.

Kanwisher, N., McDermott, J., \& Chun, M. M. (1997). The fusiform face area: A module in human extrastriate cortex specialized for face perception. Journal of Neuroscience, 17, 4302-4311.

Lavie, N. (1995). Perceptual load as a necessary condition for selective attention. Journal of Experimental Psychology: Human Perception and Performance, 21, 451-468.

Lavie, N., \& Tsal, Y. (1994). Perceptual load as a major determinant of the locus of selection in visual attention. Perception \& Psychophysics, 56, 183-197.

Liu, J., Higuchi, M., Marantz, A., \& Kanwisher, N. (2000). The selectivity of the occipitotemporal M170 for faces. Neuroreport, 11, 337-341.

Luck, S. J., Chelazzi, L., Hillyard, S. A., \& Desimone, R. (1997). Neural mechanisms of spatial selective attention in areas V1, V2, and V4 of macaque visual cortex. Journal of Neurophysiology, 77, 24-42.

Luck, S. J., \& Ford, M. A. (1998). On the role of selective attention in visual perception. Proceedings of the National Academy of Sciences, USA, 95, 825-830.

Lueschow, A., Sander, T., Boehm, S. G., Nolte, G., Trahms, L., Marx, P., \& Curio, G. (2000). Attention modulates inferotemporal MEG/ EEG activity differently for faces and houses [abstract]. Cognitive Neuroscience Society (p. 92). Cambridge, MA: MIT Press.

Mangun, G. R. (1995). Neural mechanisms of visual selective attention. Psychophysiology, 32, 4-18.

McCarthy, G., Puce, A., Belger, A., \& Allison, T. (1999). Electrophysiological studies of human face perception. II: Response properties of face-specific potentials generated in occipitotemporal cortex. Cerebral Cortex, 9, 431-444.

McCarthy, G., \& Wood, C. C. (1985). Scalp distributions of eventrelated potentials: An ambiguity associated with analysis of variance models. Electroencephalography and Clinical Neurophysiology, 62, 203-208.

Moran, J., \& Desimone, R. (1985). Selective attention gates visual processing in the extrastriate cortex. Science, 229, 782-784.

Nobre, A. C., Allison, T., \& McCarthy, G. (1998). Modulation of human extrastriate visual processing by selective attention to colours and words. Brain, 121, 1357-1368.
O'Craven, K. M., Downing, P. E., \& Kanwisher, N. (1999). fMRI evidence for objects as the units of attentional selection. Nature, 401, 584-587.

Picton, T. W., Bentin, S., Berg, P., Donchin, E., Hillyard, S. A., Johnson, R., Jr., Miller, G. A., Ritter, W., Ruchkin, D. S., Rugg, M. D., \& Taylor, M. J. (2000). Guidelines for using human event-related potentials to study cognition: Recording standards and publication criteria. Psychophysiology, 37, 127-152.

Puce, A., Allison, T., Gore, J. C., \& McCarthy, G. (1995). Face-sensitive regions in human extrastriate cortex studied by functional MRI. Journal of Neurophysiology, 74, 1192-1199.

Puce, A., Allison, T., \& McCarthy, G. (1999). Electrophysiological studies of human face perception. III: Effects of top-down processing on face-specific potentials. Cerebral Cortex, 9, 445-458.

Recanzone, G. H., \& Wurtz, R. H. (2000). Effects of attention on MTand MST neuronal activity during pursuit initiation. Journal of Neurophysiology, 83, 777-790.

Reynolds, J. H., Pasternak, T., \& Desimone, R. (2000). Attention increases sensitivity of V4 neurons. Neuron, 26, 703-714.

Ruchkin, D. S., Johnson, R., Jr., \& Friedman, D. (1999). Scaling is necessary when making comparisons between shapes of event-related potential topograhies: A reply to Haig et al. Psychophysiology, 36, 832-834.

Sams, M., Hietanen, J. K., Hari, R., Ilmoniemi, R. J., \& Lounasmaa, O. V. (1997). Face-specific responses from the human inferior occipitotemporal cortex. Neuroscience, 77, 49-55.

Sander, T. H., Wübbeler, G., Lueschow, A., Curio, G., \& Trahms, L. (2002). Cardiac artifact susbspace identification and elimination in cognitive MEG data using time-delayed decorrelation. IEEE Transactions on Biomedical Engineering, 49, 345-354.

Seidemann, E., \& Newsome, W. T. (1999). Effect of spatial attention on the responses of area MT neurons. Journal of Neurophysiology, 81, 1783-1794.

Sergent, J., Ohta, S., \& MacDonald, B. (1992). Functional neuroanatomy of face and object processing. A positron emission tomography study. Brain, 115, 15-36.

Swithenby, S. J., Bailey, A. J., Brautigam, S., Josephs, O. E., Jousmaki, V., \& Tesche, C. D. (1998). Neural processing of human faces: A magnetoencephalographic study. Experimental Brain Research, 118, 501-510.

Torriente, I., Valdes-Sosa, M., Ramirez, D., \& Bobes, M. A. (1999). Visual evoked potentials related to motion-onset are modulated by attention. Vision Research, 39, 4122-4139.

Treisman, A. M., \& Gelade, G. (1980). A feature-integration theory of attention. Cognitive Psychology, 12, 97-136.

Treue, S., \& Maunsell, J. H. (1996). Attentional modulation of visual motion processing in cortical areas MT and MST. Nature, 382, 539-541.

Urbach, T. P., \& Kutas, M. (2002). The intractibility of scaling scalp distributions to infer neuroelectric sources. Psychophysiology, 39, 791-808.

Uusitalo, M. A., \& Ilmoniemi, R. J. (1997). Signal-space projection method for separating MEG or EEG into components. Medical \& Biolocial Engineering \& Computing, 35, 135-140.

Watanabe, S., Kakigi, R., Koyama, S., \& Kirino, E. (1999). Human face perception traced by magneto- and electro-encephalography. Brain Research Cognitive Brain Research, 8, 125-142.

Watanabe, S., Kakigi, R., \& Puce, A. (2003). The spatiotemporal dynamics of the face inversion effect: A magneto- and electroencephalographic study. Neuroscience, 116, 879-895.

Wojciulik, E., Kanwisher, N., \& Driver, J. (1998). Covert visual attention modulates face-specific activity in the human fusiform gyrus: fMRI study. Journal of Neurophysiology, 79, 1574-1578.

(Received February 18, 2003; AcCePTED September 30, 2003) 